\title{
Robust Track-Following Controller Design for Hard Disk Drives With Irregular Sampling
}

\author{
Behrooz Shahsavari ${ }^{1}$, Richard Conway ${ }^{1}$, Ehsan Keikha ${ }^{2}$, Fu Zhang ${ }^{1}$, and Roberto Horowitz ${ }^{1}$ \\ ${ }^{1}$ Department of Mechanical Engineering, University of California, Berkeley, CA 94720 USA \\ ${ }^{2}$ Department of Electrical and Computer Engineering, National University of Singapore, 117576 Singapore
}

\begin{abstract}
This paper considers robust controller design for track-following in hard disk drives (HDD) with irregular sampling of the position error signal (PES) but regular (clock-driven) control updates. This sampling and actuation behavior is modeled by applying a novel discretization method to a continuous-time model of an HDD, resulting in a discrete-time linear periodically time-varying model. Then, the controller design is performed using optimal $H_{\infty}$ control for periodic systems and uses a generalization of the disk margin to quantify the robustness of the closed-loop system. To show the effectiveness of the proposed method, the design methodology is applied to a hard disk drive model and the resulting controller is validated by examining its nominal performance in terms of the root mean square of the standard deviation of the PES and robustness in terms of disk margin. Since the proposed controller has too many parameters to be implementable on an HDD due to memory limitations, we use a vector quantization method to approximate the entire parameter set of the designed controller by a smaller set of parameters.
\end{abstract}

Index Terms-Hard disk drives (HDD), $H_{\infty}$ control, irregular sampling, periodic systems, robust control.

\section{INTRODUCTION}

$\mathbf{S}$ AMPLING time in hard disk drive (HDD) servo systems is not always constant over a revolution of the disk. The position of the read/write head is attained when it passes over the servo sectors written on the disk [1]. Hence, when the servo sectors are not placed equidistantly on the tracks the sampling will be time varying on one revolution of the disk. However, since the disk is rotating, the irregularity in sampling intervals will be $N$-periodic, where $N$ is the number of servo sectors on each track. There are different factors that can make the sampling time irregular. For instance, when the center of servo tracks does not exactly coincide with the center of disk rotation, there can be large variation in the sampling rate. Another factor resulting in an irregular sampling rate is the existence of missing sectors as a result of false PES demodulation [2]. Furthermore, during the manufacturing and testing processes of some HDDs, PES sampling rates can be more than ten times that of the normal servo loop bandwidth [3] and, because track accuracy is not strongly gated, this may result in the servo system missing half of the PES samples.

When HDDs areal density is low, ignoring the variation in the sampling rate and using a linear time invariant (LTI) controller usually results in acceptable performance and robustness [4]. However, the areal density of HDDs has had $40 \%$ annual increase during the last six years and is expected to reach 2.5 $\mathrm{Tb} / \mathrm{in}^{2}$ by 2014 , which will require writing data on $19 \times 13.5$ $\mathrm{nm}^{2}$ bit cells [5]. For this high areal density, ignoring the sampling time irregularity and utilizing an LTI controller might not lead to the desired level of performance and robustness [6]. Therefore, a design methodology more carefully dealing with this irregularity in sampling rate is necessary. Two meaningful

Manuscript received December 02, 2012; revised March 30, 2013; accepted April 10, 2013. Date of current version May 30, 2013. Corresponding author: B. Shahsavari (e-mail: behrooz@berkekey.edu).

Color versions of one or more of the figures in this paper are available online at $\mathrm{http} / /$ ieeexplore.ieee.org.

Digital Object Identifier 10.1109/TMAG.2013.2259223 schemes for updating the control signal can be considered for a system with irregular sampling [6]. In the first scheme, the controller is event-driven, i.e., the control is updated as quickly as possible after receiving a new measurement. In the second scheme, the controller is clock-driven and the control action is updated at a regular rate regardless of the sampling time variation. As illustrated in [6], a system with irregular but periodic sampling time can be modeled as a linear periodically timevarying (LPTV) system and exploiting the latter scenario results in better limits of performance in such a system. Moreover, since the control action is usually passed through one or more notch filters before being applied to the actuator(s), in order to prevent the excitation of actuator high frequency resonance modes [7], the second control scheme is more attractive because it decouples the design of the notch filters from the sampling rate variation. Due to these two significant advantages, our design methodology is based on the latter control scheme. Using this control method, there might be arbitrary number of measurements between two successive control updates [8]. In Section III it is presented how our proposed controller accommodates the varied number of measurements between successive control updates.

It is worth noting that this type of servo mechanism can be considered as a network control system (NCS) with two main sources of delay. Firstly, updating the control signal may be computationally expensive and time consuming. Secondly, since the control update and sampling time are not synchronized, there usually exists a time difference between the measuring instants and the scheduled control update instants. NCSs with event-driven (irregular) control are considered in [9], where it is assumed that the control is updated at the sampling instants. The same control update scheme for NCSs when the control is updated with a delay after each sampling is considered in [10]. In both of these works it is assumed that the sampling is clock-driven and the case when the feedback signal arrives irregularly is not considered.

Performance and robustness are the most important specifications that should be considered in control design for HDDs. 
In particular, since the resulting controller must achieve a high level of performance when applied to any unit in the product line, it must have sufficient robustness margins. Traditional $H_{\infty}$ control design techniques for linear time invariant (LTI) systems can attenuate error well even under a set of plant variations [11]. One goal of this paper is to extend the $H_{\infty}$ control design methodology to systems with periodic irregular sampling. In [12], the $H_{\infty}$ control problem for an HDD with irregular sampling rate caused by missing PES data is considered. The time interval between any two subsequent successful measurements in [12] is a positive integer multiplier of the nominal sampling time, while in this work it can be any positive value. Therefore, the control design proposed in that work can only be exploited for a specific type of sampling time irregularity (e.g., the results of [12] cannot be utilized for a HDD having disk eccentricity, in which the sampling time is a trigonometric function of time). To quantify the robustness of our closed-loop system, which will be modeled as an LPTV system, we extend the idea of disk margins [13] to LPTV systems by proposing a novel idea based on $H_{\infty}$ norm of a modified open loop system. We then maximize the disk margin of the closed loop system by choosing a few shaping functions and solving an optimal $H_{\infty}$ control problem for the LPTV model of the servo system.

It is illustrated in [14] that almost all disturbances in higher $\mathrm{r} / \mathrm{min}$ HDDs can be reduced by sealing the drive and filling it with a light weight gas (e.g., Helium). This advantage of using light weight gases has guided the HDD industry toward producing Helium-filled drives. The $H_{\infty}$ control problem presented in this work includes performance weighting functions which are usually chosen based on the system noise model. Therefore, the proposed control design method can be exploited for the next generation of HDDs by knowing basic information about their noise model. Furthermore, it is shown in the paper that the complexity of the controller depends on the complexity of the performance weighting functions. Hence, using light weight gases can benefit this control design since smaller disturbances can be ignored or modeled with simpler dynamics.

\section{Modeling OF HDD}

In this section, we will describe a sampling and actuation scheme presented in [6], in which the time interval between two subsequent successful measurements is time varying while the control update rate is constant (i.e., the controller is clockdriven). Since the servo sectors are written during the manufacturing process, the time interval between reading any two particular consecutive servo sectors is constant, but it changes from sector to sector. Hence, the time intervals between two consecutive samplings can be known and used in control design. Throughout this section, the continuous-time state space model of the system is given by

$$
\begin{aligned}
\dot{x}_{c}(t) & =A_{c} x_{c}(t)+B_{c} u_{c}(t) \\
y_{c}(t) & =C_{c} x_{c}(t)+D_{c} u_{c}(t) .
\end{aligned}
$$

The signals, $x_{c}(t), u_{c}(t)$, and $y_{c}(t)$, respectively, have dimen$\operatorname{sion} n_{x}, n_{u}$, and $n_{y}$. We assume that an event-driven zero-order holder $(\mathrm{ZOH})$ is used to hold the output of the discrete-time controller for the plant. For a positive real value of period, say $T$, the state dynamics matrices for the discrete time system with period $T$ are

$$
A_{d}(T)=e^{A_{c} T}, \quad B_{d}(T)=\left(\int_{0}^{T} e^{A_{c} \tau} d \tau\right) B_{c} .
$$

We denote the time that the control signal will be updated at time step $k$ as $t_{u, k}$. Assuming that the control update rate is regular with period $T$, and $t_{u, 0}=0$, the control update instances, $t_{u, k}$, can be characterized as

$$
t_{u, k}=k T, \quad k \in \mathbb{Z} .
$$

The computational delay associated with the controller will be denoted as $\delta$, and we will assume for simplicity that $\delta<$ $T$. In particular, since the controller has to update the control signal at scheduled moments rather than as soon as receiving a feedback signal, we need to treat the computational delay as the constraint that the controller cannot use the measurements arriving in the time interval $\left(t_{u, k}-\delta, \infty\right)$ in the calculation of $u_{k}$. Equivalently, the value of $u_{k}$ will be updated at time step $k+1$ by measurements that arrive in the time interval

$$
S_{k}:=\left(t_{u, k}-\delta, t_{u, k+1}-\delta\right] .
$$

We now define

$$
\begin{aligned}
& x_{k}:=x_{c}\left(t_{u, k}\right) \\
& u_{k}:=u_{c}\left(t_{u, k}\right) .
\end{aligned}
$$

Since the controller is clock-driven and it updates the control signal regularly, we can discretized the state dynamics of (1) as

$$
x_{k+1}=A_{d}(T) x_{k}+B_{d}(T) u_{k} .
$$

It is noteworthy that the state dynamics of the discrete-time system is time invariant. In other words, since the states and control of the system are updated regularly in time, regardless of the irregularity in the sampling time, the matrices representing the state dynamics are time-invariant. It will be shown later that this LTI dynamics results in requiring less memory for storing the controller parameters. We now find the representation of a measurement at a time instant $\bar{t} \in S_{k}$. There are two cases to consider. The first case corresponds to $\bar{t} \geq t_{u, k}$. In this case, we obtain

$$
\begin{aligned}
y(\bar{t}) & =C_{c} x_{c}(\bar{t})+D_{c} u_{c}(\bar{t}) \\
& =\left[C_{c} A_{d}\left(\bar{t}-t_{u, k}\right)\right] x_{k}+\left[C_{c} B_{d}\left(\bar{t}-t_{u, k}\right)+D_{c}\right] u_{k} .
\end{aligned}
$$

The second case corresponds to $\bar{t}<t_{u, k}$. In this case, we note that

$$
\begin{aligned}
x_{c}(\bar{t})= & A_{d}\left(\bar{t}-t_{u, k-1}\right) x_{k-1}+B_{d}\left(\bar{t}-t_{u, k-1}\right) u_{k-1} \\
= & A_{d}\left(\bar{t}-t_{u, k-1}\right) A_{d}^{-1}(T)\left[x_{k}-B_{d}(T) u_{k-1}\right] \\
& +B_{d}\left(\bar{t}-t_{u, k-1}\right) u_{k-1} .
\end{aligned}
$$


For notational convenience, we define

$$
\begin{aligned}
& \bar{A}:=A_{d}\left(\bar{t}-t_{u, k-1}\right) A_{d}^{-1}(T) \\
& \bar{B}:=B_{d}\left(\bar{t}-t_{u, k-1}\right)-A_{d}\left(\bar{t}-t_{u, k-1}\right) A_{d}^{-1}(T) B_{d}(T)
\end{aligned}
$$

so that the previous expression can be written as

$$
x_{c}(\bar{t})=\bar{A} x_{k}+\bar{B} u_{k-1} .
$$

This yields

$$
\begin{aligned}
y(\bar{t}) & =C_{c} x_{c}(\bar{t})+D_{c} u_{c}(\bar{t}) \\
& =C_{c} \bar{A} x_{k}+C_{c} \bar{B} u_{k-1}+D_{c} u_{k} .
\end{aligned}
$$

Since this expression depends on $u_{k-1}$, we need to augment the state vector, i.e., we write the state dynamics of the discrete-time system as

$$
\begin{aligned}
{\left[\begin{array}{c}
x_{k+1} \\
u_{k}
\end{array}\right] } & =\hat{A}\left[\begin{array}{c}
x_{k} \\
u_{k-1}
\end{array}\right]+\hat{B} u_{k} \\
\hat{A} & :=\left[\begin{array}{cc}
A_{d}(T) & 0 \\
0 & 0
\end{array}\right], \quad \hat{B}:=\left[\begin{array}{c}
B_{d}(T) \\
I
\end{array}\right] .
\end{aligned}
$$

Again, these dynamics are LTI and do not depend on the measurement characteristics of the system. The output at time instant $\bar{t} \in S_{k}$ corresponds to

$$
y(\bar{t})=\hat{C}(k)\left[\begin{array}{c}
x_{k} \\
u_{k-1}
\end{array}\right]+\hat{D} u_{k}
$$

where

$$
\begin{aligned}
& \hat{C}(k)= \begin{cases}{\left[C_{c} \bar{A}, C_{c} \bar{B}\right],} & \bar{t} \in\left(t_{u, k}-\delta, t_{u, k}\right) \\
{\left[C_{c} A_{d}\left(\bar{t}-t_{u, k}\right), 0\right],} & \bar{t} \in\left[t_{u, k}, t_{u, k+1}-\delta\right]\end{cases} \\
& \hat{D}(k)=\left\{\begin{array}{ll}
D_{c}, & \bar{t} \in\left(t_{u, k}-\delta, t_{u, k}\right) \\
C_{c} B_{d}\left(\bar{t}-t_{u, k}\right)+D_{c}, & \bar{t} \in\left[t_{u, k}, t_{u, k+1}-\delta\right]
\end{array} .\right.
\end{aligned}
$$

Although (13) and (14) describe the output at an arbitrary time instance, these relationships do not fully describe the system output corresponding to a given time index. In particular, since there is no fixed relationship between the times at which measurements are obtained and the times at which the control is updated, the number of samples in the time interval $S_{k}$ is not necessarily constant over $k$. For simplicity, we will consider a situation in which 0,1 , or 2 measurements may be made in any time interval $S_{k}$. We thus have three cases to consider. In all three cases, we will force the discrete-time model to have two outputs. We begin by considering the case when, for a particular value of $k$, there are two measurements made in the time interval $S_{k}$; we denote the time instances corresponding to these measurements as $\overline{t_{1}}$ and $\overline{t_{2}}$. In this case, we choose

$$
y_{k}=\left[\begin{array}{l}
y\left(\overline{t_{1}}\right) \\
y\left(\overline{t_{2}}\right)
\end{array}\right] \text {. }
$$

Note that $y_{k}$ captures all of the information that the controller can use to update the value of $u_{k}$ to $u_{k+1}$. We now consider the case when, for a particular value of $k$, there are no measurements made in the time interval $S_{k}$. In this case, the controller should accept no inputs at time step $k$. Equivalently, the input into the controller should be zero. This motivates choosing

$$
y_{k}=\left[\begin{array}{l}
0 \\
0
\end{array}\right] \text {. }
$$

Choosing this form for $y_{k}$ allows the system to have a timeinvariant structure; $y_{k}$ is acting here as a placeholder so that the discrete-time model has two outputs, even at time steps when the controller has no inputs. Finally, we consider the case when, for a particular value of $k$, there is one measurement made in the time interval $S_{k}$; we denote the time instance corresponding to this measurement as $\bar{t}$. In this case, we choose

$$
y_{k}=\left[\begin{array}{c}
y(\bar{t}) \\
0
\end{array}\right] \text {. }
$$

As in the previous case, we are using zeros as placeholders so that the discrete-time model has two outputs, even at time steps when the controller only has one input. Under the assumption that the sampling of the system is periodic, (11)-(17) define an LPTV discrete-time state space model. This model describes the continuous-time model (1)-(2) under a $\mathrm{ZOH}$ on the input with sampling and actuation conditions described at the beginning of this section. However, there is one subtle detail that remains: with the convention we have chosen for defining $y_{k}$, we cannot use any arbitrary control scheme to control this model. In particular, for continuous-time causality to hold, $u_{k}$ can only depend on, $y_{k-1}, y_{k-2}, \ldots, y_{0}$, i.e., a discrete-time controller satisfies continuous-time causality if and only if it is strictly causal in the discrete-time domain.

\section{Control Design}

In this section, we present an optimal $H_{\infty}$ control design for an HDD model that is discretized by using the method discussed in Section II. Our control objective throughout this paper is to maximize the stability margins while keeping the position error signal (PES) as small as possible, in order to achieve high areal densities and low-readout error rates. We first define the metrics for performance evaluation of an LPTV system and specifically an HDD that is discretized as an LPTV system and then propose a method for robustness evaluation of such a system. Finally, the architectures used for control design and performance calculation are presented.

\section{A. Performance Analysis}

If the entire system is adequately modeled as a stochastic system, i.e., all external disturbances can be considered as random signals with Gaussian distribution, the tracking performance is normally characterized by the $3 \sigma$ value of the PES [11]. When all the disturbance sources are normalized through proper weighting functions, this root-mean-square (RMS) value is then equivalent to the $\mathrm{H}_{2}$ norm of the transfer function from those normalized disturbances to the PES, i.e.,

$$
\operatorname{RMS}(\mathrm{PES})=\left\|G_{d \rightarrow \mathrm{PES}}\right\|_{2}
$$

where $G_{d \rightarrow \text { PES }}$ is the transfer function from the Gaussian input disturbance to the PES. For an LPTV system although the output signals will not be stationary, the second-order statistics will be 


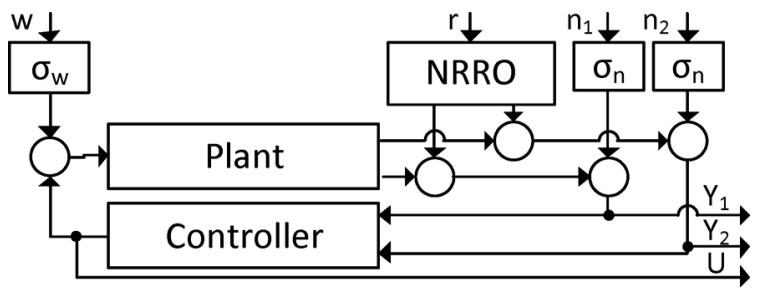

Fig. 1. Closed loop system for performance calculation.

periodic, with period $N$. Hence, the $3 \sigma$ value of PES at each sector of a HDD modeled as a LPTV system is constant and can be computed by

$$
\sigma_{k}(\mathrm{PES})=\left\|G_{d \rightarrow \mathrm{PES}}^{k}\right\|_{2}, \quad k \in\{1, \ldots, N\}
$$

where $G_{d \rightarrow \text { PES }}^{k}$ is the closed loop system from $N$ input disturbance vectors during one revolution to the PES at sector $k$. A good way to capture the performance of a controller in a HDD modeled as a LPTV system is to compute the RMS and maximum $3 \sigma$ values of PES over a revolution of disk

$$
\begin{aligned}
\operatorname{RMS}(\mathrm{PES}) & =\operatorname{RMS}_{k=1, \ldots, N}\left[\sigma_{k}(\mathrm{PES})\right] \\
\sigma_{\max } & =\max _{k \in\{1, \ldots, N\}}\left[\sigma_{k}(\mathrm{PES})\right] .
\end{aligned}
$$

Equations (18)-(19) can be computed by using the solution of a periodic Lyapunov equation. Fig. 1 shows a block diagram of the closed-loop system with all relevant disturbances. The signals $Y_{1}, Y_{2}, U, r, w, n_{1}$ and $n_{2}$ are, respectively, first and second measurement signals, control signal, independent white noises with unit variance that respectively model the effect of the nonrepeatable runout (NRRO), windage, and measurement noise for the two measurement signals. NRRO is the random lateral movement of the disk caused by the mechanical contacts in the bearing motor, and windage is the off track motion at the head caused by the turbulent nature of the air between the disk and the actuator [1]. The NRRO, $\sigma_{n}$ and $\sigma_{w}$ blocks in Fig. 1 are, respectively, the NRRO model, the standard deviation of measurement noise, and the standard deviation of the windage. We use the same idea as Section II to discretize the NRRO model, i.e., we find a continuous-time model for the NRRO and then discretize it as a LPTV system. Accordingly, the NRRO model has two outputs similar to the plant model.

\section{B. Control Design}

To quantify the robustness of the closed-loop systems, we would like to use gain and phase margins. However, since computing the gain and phase margins of an LPTV system is difficult (or impossible), we quantify the robustness of the system by generalizing an idea known as disk margin [13]. For LTI systems, the disk margin is a more rigorous measure of robustness than gain and phase margins because it quantifies how close the open-loop frequency response (e.g., the Nyquist plot) comes to -1 in all directions, rather than just along the real axis and the unit circle (as quantified respectively by the gain and phase margins). For a particular plant and a designed controller the disk margin can be found by adding a new input and output signal to the system and calculating the $H_{\infty}$ norm from the new input

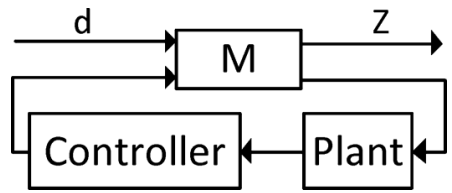

Fig. 2. Modified close loop system for disk margin calculation.

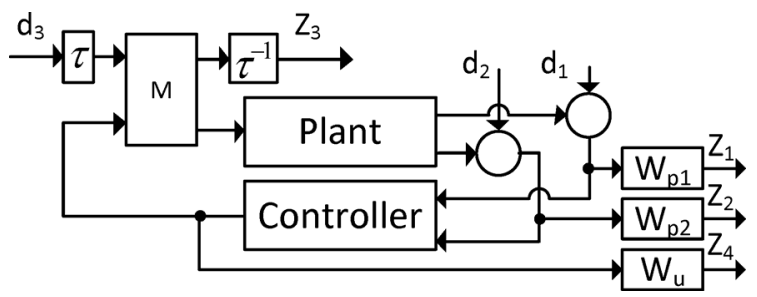

Fig. 3. Block diagram for control design.

to the new output channel. This idea is shown in Fig. 2. In this figure, the block $M$ is a matrix given by

$$
M:=\left[\begin{array}{cc}
1 & \sqrt{2} \\
\sqrt{2} & 1
\end{array}\right] .
$$

This matrix $M$ is included in the block diagram so that the $H_{\infty}$ norm from $d$ to $Z$ represents the disk margin of the closed-loop system by the following relations:

$$
\begin{aligned}
& \mathrm{DGM}= \begin{cases}\infty & \beta \leq 1 \\
(\beta+1) /(\beta-1) & 1<\beta<\infty \\
1 & \beta=\infty\end{cases} \\
& \mathrm{DPM}= \begin{cases}\frac{\pi}{2} & \beta \leq 1 \\
\cos ^{-1}\left(\frac{\beta^{2}-1}{\beta^{2}+1}\right) & 1<\beta<\infty \\
0 & \beta=\infty\end{cases}
\end{aligned}
$$

where DGM, DPM and $\beta$ are, respectively, the disk gain and phase margins of the closed loop system and the $H_{\infty}$ norm from $d$ to $Z$. Since the computation of the disk margin only requires the computation of a single $H_{\infty}$ norm, the concept and method of computation easily generalize to LPTV systems.

The architecture we use for control design is shown in Fig. 3. The blocks $W_{p 1}, W_{p 2}, W_{u}$ and $\tau$ are design parameters; they are, respectively, the performance weighting functions for the sensitivity functions corresponding to the first and second measurements, a control effort weighting value, and a static uncertainty scaling parameter, as appears in the $\mathrm{D}-\mathrm{K}$ iteration heuristic for $\mu$-synthesis. Note that the $H_{\infty}$ norm from $d_{3}$ to $Z_{3}$ represents the disk margin of the closed-loop system.

According to (20) and (21), minimizing the $H_{\infty}$ norm from $d_{3}$ to $Z_{3}$ maximizes the disk margins of the closed loop system. However, we need to keep the performance of the system larger than predefined values. In other words, we want to satisfy the following two constraints:

$$
\begin{aligned}
& 3 \sigma^{\mathrm{PES}} \leq 3 \sigma^{\mathrm{req}} \\
& 3 \sigma_{\max }^{\mathrm{PES}} \leq 3 \sigma_{\text {max }}^{\mathrm{req}}
\end{aligned}
$$

where $3 \sigma^{\mathrm{PES}}, 3 \sigma_{\max }^{\mathrm{PES}}$ are the RMS and maximum $3 \sigma$ values of PES, and $3 \sigma^{\text {req }}$ and $3 \sigma_{\max }^{\text {req }}$ are the required values, e.g., the RMS and maximum $3 \sigma$ values achieved by existing controllers.

Finally, we can formulate the optimization problem that aims to maximize the robustness subject to the aforementioned constraints as 


$$
\begin{gathered}
\min _{K}\left\|G_{\mathrm{cl}}(K)\right\|_{\infty} \\
\text { subject to } \\
3 \sigma^{\mathrm{PES}} \leq 3 \sigma^{\mathrm{req}} \\
3 \sigma_{\max } \leq 3 \sigma_{\max }^{\mathrm{req}}
\end{gathered}
$$

where $K$ and $G_{\mathrm{cl}}(K)$ are the controller and the closed loop system shown in Fig. 3. Since the solution of the optimization problem strongly depends on the design parameters (e.g., weighting functions) we use the following three-step algorithm to find the desired controller.

1) Choose design parameters $\left(W_{p 1}, W_{p 2}, W_{u}\right.$ and $\left.\tau\right)$.

2) Solve (22) (ignore the constraints).

3) If (23) is

a) satisfied: terminate and use the $K$ found in 2 ;

b) not satisfied: return to step 1 .

Once proper values have been chosen for all design parameters in step 1, in step 2 the controller can be designed using optimal $H_{\infty}$ control in the following form:

$$
\begin{aligned}
& A_{K}(k)=\hat{A}-\hat{B}\left[\begin{array}{l}
L_{B 1}(k) \\
L_{B 2}(k)
\end{array}\right]-L_{c}(k) \hat{C}(k) \\
& B_{K}(k)=L_{c}(k), \quad C_{K}(k)=-L_{B 2}(k), \quad D_{K}(k)=0
\end{aligned}
$$

where $A_{K}(k), B_{K}(k), C_{K}(k)$ and $D_{K}(k)$ are state space matrices of the controller. Matrices $L_{B 1}(k), L_{B 2}(k)$ and $L_{c}(k)$ can be calculated by the solution of periodic Riccati equations [12].

\section{Controller Parameters Order Reduction}

The control synthesis algorithm described in the previous section results in a periodically time varying controller with a large period. The total number of sets of controller matrices [given in (24)] to be stored in the memory of the servo controller is equal to the number of servo sectors in one data track (mostly between 300 and 400 in current HDDs). In a physical HDD, there is a limited amount of memory available to store the controller parameters and it is almost impossible to reserve so much memory for storing all of these values. As a result, the current designed controller is not directly suitable for implementation.

In this paper we introduce two different approaches to reduce the number of controller parameters required to be stored in memory. The first approach is introducing an innovative discretization method by making the control action regular, which eliminates the time variation in controller parameters $\hat{A}$ and $\hat{B}$. The second approach is to use a vector quantization technique to approximate time-varying controller parameters by a reduced set of matrices, i.e., by replacing $\hat{C}(k), L_{B 1}(k), L_{B 2}(k)$ and $L_{c}(k)$ with approximate values.

Vector quantization is a well-known and efficient algorithm in signal processing which is based on the competitive learning paradigm. It works by dividing a large set of points (vectors) into groups. Each group is represented by its centroid point, as in k-means and some other clustering algorithms.

The basic idea of the algorithm which we have used to approximate the time varying parameters is as follows: the mean of the data set [e.g., the first column of $L_{c}(k)$ ] is obtained and is split into two points which will respectively be the centroids of two new clusters. The Euclidean distance of each point from these centroids is calculated, and each one is associated with the cluster having the closest centroid. The centroid of each cluster is subsequently replaced by the mean of the vectors in that cluster. If the total distance of vectors from the centroids of their clusters is not improved substantially, the centroids will split again. This continues until either the required number of clusters is reached or the improvement remains inadequate.

In our case we would like to get an approximation of $\hat{C}(k), L_{B 1}(k), L_{B 2}(k)$ and $L_{c}(k)$ such that the overall performance of the closed loop system remains in predefined limits. Our simulation results show that it is more effective if we apply vector quantization to each column or row of these matrices separately and combine the approximated parameters at the end.

\section{RESULTS}

This section presents numerical results to show the effectiveness of the proposed optimal $H_{\infty}$ controller for track following in an HDD with irregular sampling. Our simulation is based on a model for a single stage 3.5-in HDD.

\section{A. Plant Model}

As mentioned in Section II, the required continuous-time plant model should be discretized by the proposed method in that subsection. To obtain this continuous-time model, we first measured the discrete time frequency response of the voice-coil motor (VCM) in the aforementioned HDD, and then found a continuous-time model such that its discrete time frequency response fits the measured response. The particular setup used in this study has very regular sampling time intervals, which let us discretize the system in the fitting process by using MATLAB ${ }^{\circledR} C 2 d$ function with the $\mathrm{ZOH}$ method. It should be noticed that this discretization is just for the fitting process, and the model for control design or performance analysis should be discretized by the method presented in Section II.

The continuous-time model $G_{c}$, found by the frequency response fitting process, is in the form of

$$
G_{c}=G_{e}\left(\frac{1}{s^{2}}+G_{r 1}+\cdots+G_{r 6}\right)
$$

where $G_{e}$ models a low pass filter embedded in the electronic circuit of the servo amplifier part. $G_{r 1}$ to $G_{r 6}$ captures the first six important second-order resonance modes appeared in the frequency response, i.e.,

$$
G_{r i}=\frac{g_{i}}{s^{2}+2 \zeta \omega s+\omega^{2}}, \quad i=1, \ldots, 6 .
$$

In order to validate the accuracy of the continuous-time plant model used for the chosen HDD, we compared the $3 \sigma$ value of PES computed based on real PES measured in the HDD with the corresponding value simulated based on our plant model. Since we had access to the PES during track following in this particular setup, we were able to find the $3 \sigma$ value of PES in this HDD. 


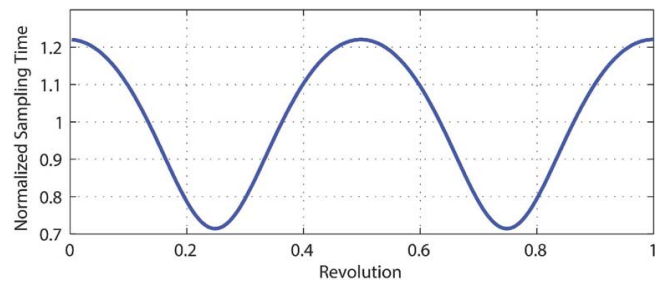

Fig. 4. Normalized sampling time in one revolution.

Due to disturbances during servo track writing, the created reference for the track-center deviates from being perfectly circular in shape. These deviations in the track reference constitute the repeatable runout (RRO) for the head positioning servo mechanism. We measured PES at 32000 consecutive sectors during track following and computed the RRO at each sector by averaging all of the PES values measured at that sector. Once the RRO was computed, we subtracted it from the PES data to form the NRRO and then found the $3 \sigma$ value of the NRRO. For this particular setup, this value was $4.95 \%$ of the track width. Since we are interested in comparing the actual $3 \sigma$ value with the simulated one based on our plant model, we used the track-following controller provided by our industry partner to calculate the $3 \sigma$ value of the closed-loop PES. This value in our model is $8 \%$ less than the value calculated based on measurement, which validates the accuracy of our model. We used the ratio of the simulated to measured performance, which is 0.92 , to compensate the plant model inaccuracy in the performance analysis. Finally, we discretized $G_{c}$ by the proposed method in Section II.

\section{B. Eccentricity and Limit of Performance}

As mentioned before, if the center of data tracks, disk and rotation are not exactly coincident, the sampling time over one revolution of the disk will be varying. It is easy to show that when the servo tracks are written radially on the disk and the data tracks are concentric circles having a center coinciding with the rotation center, the time interval between the $i$ th and $(i+1)$ th sampling, denoted as $\Delta t_{i}$, can be calculated by the following two relations:

$$
\begin{aligned}
\Delta t_{i} & =\left(\theta_{i+1}-\theta_{i}\right) / \omega, \quad i \in 0, \ldots, N-1 \\
\cos \left(\theta_{i}\right) & =\cos \left(\frac{2 \pi i}{N}\right) \sqrt{1-\left(e \sin \left(\frac{2 \pi i}{N}\right)\right)^{2}}
\end{aligned}
$$

where $e$ and $\omega$ are, respectively, the normalized eccentricity between the disk and rotation centers, and the rotation speed. We use $e=0.7$ to calculate the sampling time interval vector. Although this eccentricity ratio is very large, we use it to show the effectiveness of the proposed methods even though the sampling time intervals vary significantly. For this particular chosen value, the peak to peak variation of sampling time is equal to $50 \%$ of the average value. Fig. 4 shows the sampling time vector when it is normalized by the nominal sampling time, which is equal to the average value of the irregular sampling times.

Two of the required parameters to perform our control design are $\sigma^{\text {req }}$ and $\sigma_{\max }^{\text {req }}$ in (23). To choose reasonable values for them, we first determined the limits of performance for this particular setup as discussed in [6]. We then chose $\sigma^{\mathrm{req}}$ and $\sigma_{\max }^{\mathrm{req}}$ as $15 \%$ larger than their corresponding limits.
TABLE I

CONTROL Signal AND PES PERformances

\begin{tabular}{ccccc}
\hline \hline $\begin{array}{c}\text { Controller } \\
\text { Type }\end{array}$ & $3 \sigma^{u}$ & $3 \sigma_{\max }^{u}$ & $3 \sigma^{P E S}(\mathrm{~nm})$ & $3 \sigma_{\max }^{\text {PES }}(\mathrm{nm})$ \\
\hline Limits of & 1.00 & 1.70 & 2.57 & 2.85 \\
Performance & & & & \\
$H_{\infty}$ controller & 1.30 & 2.21 & 2.90 & 3.04 \\
Quantized $H_{\infty}$ & 1.44 & 2.47 & 2.93 & 3.06 \\
controller & & & & \\
\hline \hline
\end{tabular}

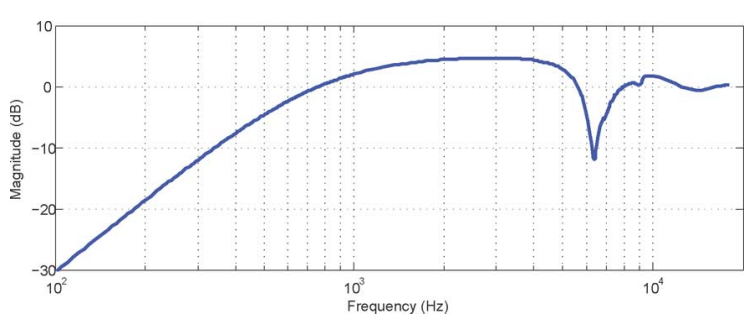

Fig. 5. Closed loop sensitivity function at the output of the controller.

The limits of performance are listed in Table I, where $3 \sigma^{u}$ and $3 \sigma_{\max }^{u}$ are, respectively, the RMS and maximum $3 \sigma$ values of control signal. These characteristics of the control signal are also metrics for evaluating controller performances.

Since the system is LPTV, frequency response methods do not apply. Despite this, we quantified the performance of the closed-loop system by using what we call an "empirical Bode magnitude plot," which uses a swept-sine approach to find an approximate frequency response magnitude. The assumption here is that inputting a sine wave into the system produces a negligible response at other frequencies. Note that for an LTI system, both empirical Bode magnitude plot and Bode magnitude plot are equal.

\section{Controller Design}

We used the architecture shown in Fig. 3 to construct the controller. According to this figure, the open loop system consists of the plant and weighting functions. Hence, the number of states needed to define this system in a state space form is equal to the summation of states of these elements. The $H_{\infty}$ optimal controller designed by the method discussed in Section II will have the same number of states as the open loop system. So, it is desirable to select weighting functions with small orders. After some iteration, we chose constant values of $W_{p 1}, W_{p 2}$ and $W_{u}$ resulting in a controller that achieved good performance in terms of the $3 \sigma$ values of PES [i.e., it satisfies (23)] and adequate robustness in terms of the disk margin. The RMS and maximum $3 \sigma$ values of the PES attained by this controller are listed in Table I. The disk margin of the closed loop system is 0.39 , which guarantees a gain and phase margin of at least 7.2 $\mathrm{dB}$ and $43^{\circ}$, respectively. Fig. 5 shows the empirical Bode magnitude plot for the sensitivity function of this system, measured at the output of the controller.

\section{Quantization of Controller Parameters}

Since our controller has a large number of time varying matrices [i.e., $\hat{C}(k), L_{B 1}(k), L_{B 2}(k)$ and $L_{c}(k)$ ], we need to use 
TABLE II

Number of Clusters for CONTROlLer PARAMETERs QUANTIZATION

\begin{tabular}{cccccc}
\hline \hline $\begin{array}{c}1^{\text {st }} \text { row } \\
\text { of } \hat{C}\end{array}$ & $\begin{array}{c}2^{\text {nd }} \text { row } \\
\text { of } \hat{C}\end{array}$ & $\begin{array}{c}\text { All rows } \\
\text { of } L_{B 1}\end{array}$ & $\begin{array}{c}\text { All rows } \\
\text { of } L_{B 2}\end{array}$ & $\begin{array}{c}1^{\text {st }} \text { col. } \\
\text { of } L_{C}\end{array}$ & $\begin{array}{c}2^{\text {nd }} \text { col. } \\
\text { of } L_{C}\end{array}$ \\
\hline 16 & 2 & 1 & 1 & 16 & 2 \\
\hline \hline
\end{tabular}

vector quantization to reduce the number of parameters, as discussed in Subsection III-C. The number of clusters was chosen such that the quantized controller satisfies the memory constraints and still has a reasonable performance. The performance of this controller and number of clusters are listed in Tables I and II, respectively. Using the novel discretization method we presented in Section II and the quantization method with the number of clusters listed in Table II, we were able to reduce the number of parameters that needed to be stored by a factor of about 181 , while the performance is reduced by only $1 \%$.

\section{CONCLUSION AND Future WORK}

In this paper, we considered robust controller design of HDDs with irregular sampling but regular control updates. We modeled this sampling and actuation behavior by applying a novel discretization method to a continuous-time model of the HDD. Once we had an appropriate discrete-time model of the HDD, we used LPTV $H_{\infty}$ control to design a robust controller. Unlike other papers using $H_{\infty}$ to design HDD controllers, we did not use a weighted uncertainty model to quantify the robustness of the closed-loop system. Instead, we used the disk margin. The benefit of using this measure of robustness is that it can be used to compute lower bounds on the gain and phase margins, thus putting our robustness margins into a framework more commonly used by the HDD industry.

Once we designed a controller, we evaluated its robustness by checking the disk margin at the control signal. We also checked the performance using a model of the system that included a detailed model of the stochastic disturbances acting on the system. The performance of the controller we designed is only $13 \%$ smaller than the limits of performance, while it achieves high level of robustness in term of disk margin.

Since the designed controller had too many parameters to be implementable on an HDD due to memory limitations, we used vector quantization to approximate the parameters of the designed LPTV controller by a smaller set of parameters. The approximate controller needs 181 times less memory and its performance is only $1 \%$ lower than the original controller. In the future, we will use the methods of [12] to do loop-shaping designs for this control architecture. This will be done by simply incorporating an appropriate frequency weighting function into our $H_{\infty}$ design to directly specify an upper bound on the closedloop sensitivity function.

\section{ACKNOWLEDGMENT}

This work was supported in part by Computer Mechanics Laboratory (CML) at the University of California, Berkeley, and the author, E. Keikha, was supported by a research grant from National Research Foundation (NRF), Singapore, under CRP Award NRF-CRP-4-2008-06.

\section{REFERENCES}

[1] A. Al Mamun, G. Guo, and C. Bi, Hard Disk Drive: Mechatronics and Control. Boca Raton, FL, USA: CRC, 2007, vol. 23.

[2] R. M. Ehrlich, "Methods for improving servo-demodulation robustness," U.S. Patent 6,943,981, Sep. 13, 2005.

[3] G. Guo and J. Yu, "Servo format trend and servo channel improvement for high areal density," in Proc. APMRC Dig., 2010, pp. 1-2.

[4] M. Hirata, K.-Z. Liu, T. Mita, and T. Yamaguchi, "Head positioning control of a hard disk drive using $H^{\infty}$ theory," in Proc. 31st IEEE Conf. Decision and Control, 1992, pp. 2460-2461, IEEE.

[5] R. E. Fontana, S. R. Hetzler, and G. Decad, "Technology roadmap comparisons for tape, hdd, and nand flash: Implications for data storage applications," IEEE Trans. Magn., vol. 48, no. 5, pp. 1692-1696, May 2012.

[6] B. Shahsavari, R. Conway, E. Keikha, and R. Horowitz, "Limits of performance in systems with periodic irregular sampling and actuation rates," in Proc. 6th IFAC Symp. Mechatronic Systems (Mechatronics'13), 2013

[7] T. Semba, N. Kagami, and A. Tokizono, "Method and apparatus for suppressing mechanical resonance in a disk drive storage device using a notch filter," U.S. Patent 6,219,196, Apr. 17, 2001.

[8] B. Shahsavari, R. Conway, E. Keikha, and R. Horowitz, "Robust control design for hard disk drives with irregular sampling," in Proc. APMRC, 2012 Dig., 2012, pp. 1-2, IEEE.

[9] M. Yu, L. Wang, and T. Chu, "Sampled-data stabilisation of networked control systems with nonlinearity," in IEE Proc.-Control Theory and Applications, 2005, vol. 152, no. 6, pp. 609-614, IET.

[10] H. Gao, T. Chen, and J. Lam, "A new delay system approach to network-based control," Automatica, vol. 44, no. 1, pp. 39-52, 2008.

[11] X. Huang and R. Horowitz, "Robust controller design of a dual-stage disk drive servo system with an instrumented suspension," IEEE Trans. Magn., vol. 41, no. 8, pp. 2406-2413, Aug. 2005.

[12] J. Nie, E. Sheh, and R. Horowitz, "Optimal h control design and implementation of hard disk drives with irregular sampling rates," IEEE Trans. Control Syst. Technol., vol. 20, no. 2, pp. 402-407, Jun. 2012.

[13] J. D. Blight, R. Lane Dailey, and D. Gangsaas, "Practical control law design for aircraft using multivariable techniques," Int. J. Contr., vol. 59, no. 1, pp. 93-137, 1994.

[14] K. Aruga, M. Suwa, K. Shimizu, and T. Watanabe, "A study on positioning error caused by flow induced vibration using helium-filled hard disk drives," IEEE Trans. Magn., vol. 43, no. 9, pp. 3750-3755, Sep. 2007. 